\title{
Cognitive impairment and risk of future stroke: a systematic review and meta-analysis
}

\author{
Meng Lee MD, Jeffrey L. Saver MD, Keun-Sik Hong MD PhD, Yi-Ling Wu MS, Hsing-Cheng Liu MD PhD, \\ Neal M. Rao MD, Bruce Ovbiagele MD MS
}

Competing interests: Keun-Sik Hong has received a grant from Pfizer Pharmaceuticals Korea; speaker fees from Pfizer Pharmaceuticals Korea, Sanofi-Aventis Korea, Bayer Korea and

Boehringer Ingelheim Korea, and consulting fees from Pfizer Pharmaceuticals Korea, Bayer Korea and Boehringer Ingelheim Korea. No competing interests were declared by the other authors.

Correspondence to: Bruce Ovbiagele, ovibes@ musc.edu

This article has been peer reviewed.

CMAJ 2014. DOI:10.1503 /cmaj.140147

\begin{abstract}
Background: Several studies have assessed the link between cognitive impairment and risk of future stroke, but results have been inconsistent. We conducted a systematic review and meta-analysis of cohort studies to determine the association between cognitive impairment and risk of future stroke.

Methods: We searched MEDLINE and Embase (1966 to November 2013) and conducted a manual search of bibliographies of relevant retrieved articles and reviews. We included cohort studies that reported multivariable adjusted relative risks and $95 \%$ confidence intervals or standard errors for stroke with respect to baseline cognitive impairment.
\end{abstract}

Results: We identified 18 cohort studies (total 121879 participants) and 7799 stroke events.

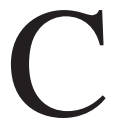
ognitive impairment is a major contributor to disability and dependence worldwide. Globally, stroke is the leading cause of long-term disability among adults and the second leading cause of death. ${ }^{1}$ The high cumulative risk of dementia or stroke or both conditions has been shown by the Framingham study, ${ }^{2}$ and the urgent need to improve knowledge regarding cognition and vascular conditions has been emphasized in a specific meeting providing harmonized standards. ${ }^{3}$ Beyond their personal tolls, both of these conditions carry substantial social and economic burdens. These conditions also correlate strongly with increasing age. Given the projected substantial rise in the number of older people around the world, prevalence rates of cognitive impairment and stroke are expected to soar over the next several decades, especially in high-income countries. ${ }^{4,5}$

Shared pathophysiologic mechanisms seem to exist between cognitive impairment and cerebrovascular disease. ${ }^{6}$ Indeed, risk factors for stroke (hypertension, hyperlipidemia, diabetes, obesity and physical inactivity) have been shown to play a role in the onset and progression of cognitive
Pooled analysis of results from all studies showed that stroke risk increased among patients with cognitive impairment at baseline (relative risk [RR] 1.39, 95\% confidence interval $[\mathrm{Cl}] 1.24-1.56)$. The results were similar when we restricted the analysis to studies that used a widely adopted definition of cognitive impairment (i.e., Mini-Mental State Examination score $<25$ or nearest equivalent) (RR 1.64, 95\% Cl 1.46-1.84). Cognitive impairment at baseline was also associated with an increased risk of fatal stroke (RR $1.68,95 \% \mathrm{Cl}$ 1.21-2.33) and ischemic stroke (RR 1.65, 95\% Cl 1.41-1.93).

Interpretation: Baseline cognitive impairment was associated with a significantly higher risk of future stroke, especially ischemic and fatal stroke.

impairment, ${ }^{7}$ and it is well established that stroke itself increases the risk of future cognitive impairment. ${ }^{8}$ However, whether cognitive impairment increases the risk of future stroke remains unclear. Early identification and regular surveillance for cognitive impairment could potentially enable prompt initiation of treatment aimed at not only potentially limiting further deterioration of cognitive function (if mild), but also possibly reducing the risk of future stroke through timely and optimal control of risk factors.

Several published studies have assessed the association between cognitive impairment and subsequent risk of stroke, but the results have not been consistent. We performed a systematic review and meta-analysis to determine the qualitative and quantitative association between baseline cognitive impairment and risk of future stroke.

\section{Methods}

\section{Search strategy}

Our search strategy was based on the recommendations of the Meta-analysis of Observational Studies in Epidemiology group. ${ }^{9}$ We searched MEDLINE via PubMed (1966 to November 
2013) and Embase (1966 to November 2013) using the following search strategy: stroke OR cerebrovascular disease OR cerebrovascular attack AND cognitive impairment OR memory impairment OR dementia OR Alzheimer disease AND cohort OR follow-up OR prospective OR trial OR incidence OR incident. No language restrictions were applied. Further information was retrieved through a manual search of references from relevant published original studies and reviews. ${ }^{10}$

\section{Study selection and quality assessment}

We included studies if they were cohort studies (prospective or retrospective); evaluated cognitive function at baseline; assessed stroke event as an outcome during the follow-up period (e.g., if a person had a stroke before enrolment, the stroke event during follow-up would be recurrent stroke; if a person did not have a stroke before enrolment, the stroke event during follow-up would be first stroke event); had an intended follow-up period of at least 1 year for all participants; and reported quantitative estimates of the multivariable adjusted relative risk (RR) and 95\% confidence interval (CI) or standard error for the log RR for future stroke associated with cognitive impairment at baseline. We excluded studies if they had a cross-sectional or casecontrol design, had a majority of participants with stroke at baseline, reported only unadjusted or age- and sex-adjusted RRs or were duplicate reports.

We extracted the following information from the studies: first author's name, study name, publication year, study country, number of followup years, number of participants (total and number with cognitive impairment), mean age of participants, percentage of women, method used to assess cognitive impairment, stroke outcome (total, fatal, nonfatal, ischemic, hemorrhagic), number of stroke events and adjusted covariates included in the models of analysis. Two of us (M.L. and K.S.H.) independently extracted data from eligible studies. Discrepancies were resolved by discussion with a third investigator (B.O.) and by referencing the original report.

The quality of studies was assessed using criteria modified from a previous meta-analysis, ${ }^{10}$ with consideration of the following aspects: study design, maintenance of comparable groups, length of follow-up, maximal adjustment for potential confounders, exclusion of participants with baseline stroke, total stroke reported (not just ischemic or fatal stroke) and generalizability to other populations. Studies were graded as high quality if they met at least 5 of the 7 criteria and low quality if they met fewer than 5 .

\section{Data synthesis}

We used multivariable adjusted outcome data (expressed as RRs and 95\% CIs or standard errors). When studies provided estimates for cognitive impairment based on more than one assessment method, we used estimates from the Mini-Mental State Examination, if available, for our primary analysis. In each study, we converted these values by using their natural logarithms, and we calculated the standard errors from these logarithmic numbers and their corresponding $95 \%$ CIs.

For the statistical analysis, we combined log RRs and standard errors using the inverse variance approach. We used a random-effects model and explored for sources of inconsistency $\left(I^{2}\right)$ and heterogeneity. A fixed-effects model was used for comparison with the random-effects model on the overall risk estimate. Reported $p$ values were 2-sided, with significance set at less than 0.05 . Heterogeneity was assessed by the $p$ value of $\chi^{2}$ statistics and by the $I^{2}$ statistic, which describes the percentage of variability in the effect estimates that is due to heterogeneity rather than to chance. ${ }^{11,12}$ Based on the suggestion of the Cochrane Collaboration, we regarded heterogeneity as possibly unimportant when the $I^{2}$ value was less than $40 \%$ and high when it was more than $75 \% .{ }^{13}$ We assessed publication bias graphically using a funnel plot and mathematically using an adjusted rank-correlation test, according

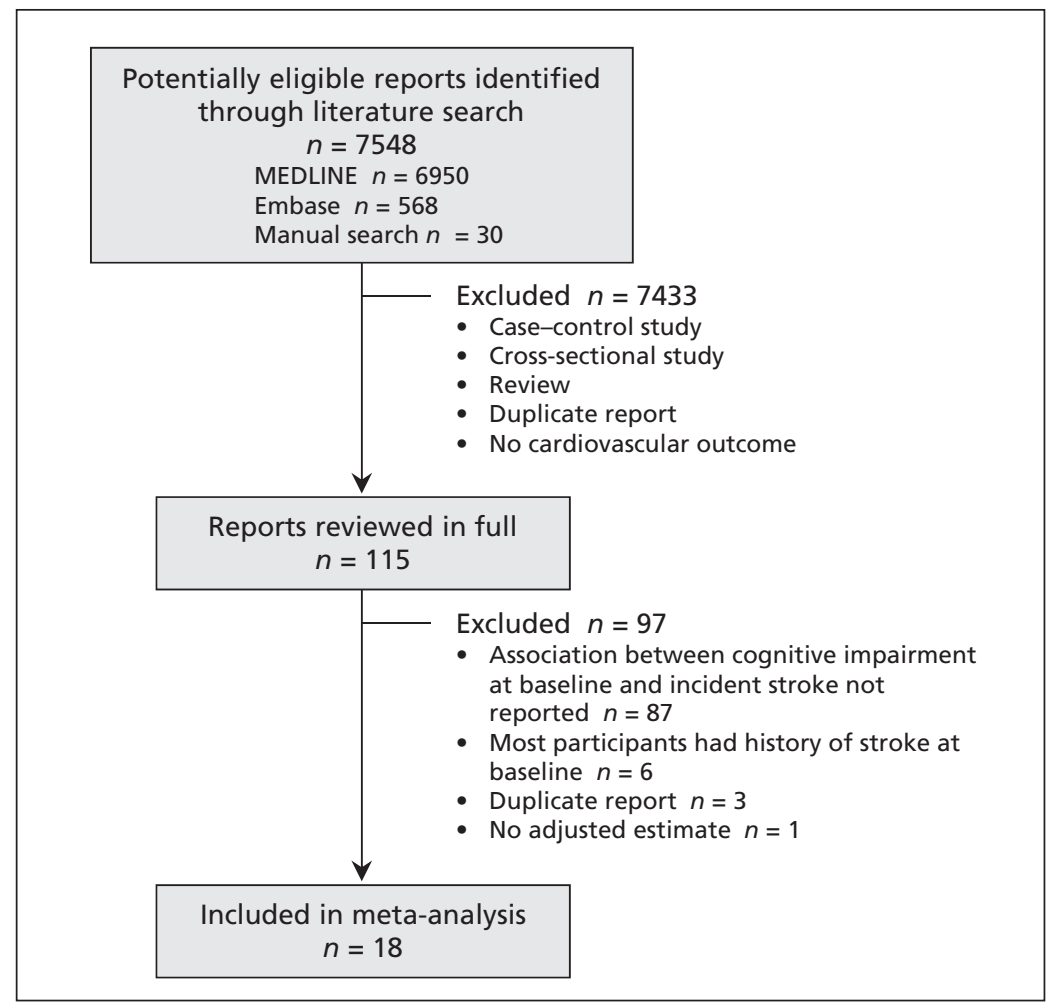

Figure 1: Selection of studies for the meta-analysis. 
Table 1: Characteristics of studies included in a meta-analysis of the association between baseline cognitive impairment and risk of future stroke

\begin{tabular}{|c|c|c|c|c|c|c|c|c|}
\hline Study & $\begin{array}{l}\text { Study } \\
\text { population }\end{array}$ & $\begin{array}{c}\text { No. of } \\
\text { patients } \\
\text { (\% female) }\end{array}$ & $\begin{array}{c}\text { Stroke } \\
\text { at } \\
\text { baseline, } \\
\%\end{array}$ & $\begin{array}{l}\text { Age, } \\
\text { yr, } \\
\text { mean }\end{array}$ & $\begin{array}{l}\text { Measures and } \\
\text { definition } \\
\text { of cognitive } \\
\text { impairment }\end{array}$ & $\begin{array}{l}\text { Duration of } \\
\text { follow-up, yr }\end{array}$ & Outcomes & $\begin{array}{l}\text { No. of } \\
\text { stroke events }\end{array}$ \\
\hline $\begin{array}{l}\text { de Moraes } \\
\text { et al.,18 2003, } \\
\text { United States }\end{array}$ & General & $11958(53)$ & 0 & 57 & $\begin{array}{l}\text { DWR; lowest } \\
\text { quartile }\end{array}$ & 6.2 & Ischemic stroke & 188 \\
\hline $\begin{array}{l}\text { Chi et al., }{ }^{19} 2013, \\
\text { Taiwan }\end{array}$ & $\begin{array}{l}\text { General; } \\
\text { propensity } \\
\text { score } \\
\text { matching }\end{array}$ & $5880(59)$ & 0 & 75 & $\begin{array}{l}\text { MMSE score 10-26 } \\
\text { and receipt of } \\
\text { ACHEI }\end{array}$ & $\begin{array}{l}4 \text { ischemic + } \\
\text { TIA; } 4.2 \\
\text { hemorrhagic }\end{array}$ & $\begin{array}{l}\text { Ischemic stroke + } \\
\text { TIA and } \\
\text { hemorrhagic } \\
\text { stroke recorded } \\
\text { separately }\end{array}$ & $\begin{array}{l}605 \text { ischemic } \\
+ \text { TIA; } 84 \\
\text { hemorrhagic }\end{array}$ \\
\hline $\begin{array}{l}\text { Clarke et al., }{ }^{20} \\
\text { 2011, Canada }\end{array}$ & $\geq 65 \mathrm{yr}$ & $9451(58)$ & 0 & 73.6 & $\begin{array}{l}\text { Dementia or CIND } \\
\text { at clinical } \\
\text { examination }\end{array}$ & 10 & $\begin{array}{l}\text { Fatal and } \\
\text { nonfatal stroke } \\
\text { recorded } \\
\text { separately }\end{array}$ & $\begin{array}{l}172 \text { fatal; } \\
701 \text { nonfatal }\end{array}$ \\
\hline $\begin{array}{l}\text { de Galan et al., }{ }^{21} \\
2009 \text {, multiple } \\
\text { countries }\end{array}$ & $\begin{array}{l}\text { Diabetes, } \\
\geq 55 \mathrm{yr}\end{array}$ & $11132(42)$ & 9.2 & 66 & $\begin{array}{l}\text { MMSE score } 24-27 \\
<24\end{array}$ & 5 & All stroke & 484 \\
\hline $\begin{array}{l}\text { Ferrucci et al..22 } \\
\text { 1996, United } \\
\text { States }\end{array}$ & $\geq 71 \mathrm{yr}$ & $5024(66)$ & 0 & 78.5 & $\begin{array}{l}\text { SPMSQ score } \\
4-6,0-3\end{array}$ & 4.3 & All stroke & 259 \\
\hline $\begin{array}{l}\text { Gale et al., }{ }^{23} \\
\text { 1996, United } \\
\text { Kingdom }\end{array}$ & $\geq 65 \mathrm{yr}$ & $921(45)$ & $N A^{*}$ & 75 & HAMT score $8-9, \leq 7$ & 20 & $\begin{array}{l}\text { Fatal ischemic } \\
\text { stroke }\end{array}$ & 162 \\
\hline $\begin{array}{l}\text { Glymour et al.., }{ }^{24} \\
\text { 2010, United } \\
\text { States }\end{array}$ & $\geq 50 \mathrm{yr}$ & $19087(59)$ & 0 & 66 & $\begin{array}{l}\text { Summing recall } \\
\leq 6 \text { (total score } 20 \text { ) }\end{array}$ & 8.1 & All stroke & 1864 \\
\hline $\begin{array}{l}\text { Liebetrau } \\
\text { et al. } 25 \text { 2008, } \\
\text { Sweden }\end{array}$ & $85 \mathrm{yr}$ & $401(70)$ & 0 & 85 & DSM III criteria & 3 & All stroke & 56 \\
\hline $\begin{array}{l}\text { O'Donnell } \\
\text { et al. } 262012, \\
\text { multiple } \\
\text { countries }\end{array}$ & $\begin{array}{l}\text { High } \\
\text { cardiovascular } \\
\text { risk }\end{array}$ & $30959(30)$ & 21 & 66.5 & $\begin{array}{l}\text { MMSE score 27-29, } \\
24-26,<24\end{array}$ & 4.7 & All stroke & 1374 \\
\hline $\begin{array}{l}\text { Ostir et al.,. }{ }^{27} \\
2003, \text { United } \\
\text { States }\end{array}$ & $\begin{array}{l}\text { Hispanic, } \\
\geq 65 \mathrm{yr}\end{array}$ & $2682(59)$ & 0 & 72 & MMSE score $<21$ & 7 & All stroke & 238 \\
\hline $\begin{array}{l}\text { Pettigrew } \\
\text { et al., } 282000, \\
\text { United States }\end{array}$ & $\begin{array}{l}\text { Asymptomatic } \\
\text { carotid } \\
\text { atherosclerosis }\end{array}$ & $1659(34)$ & 25 & 67 & $\begin{array}{l}\text { MMSE score 25-27, } \\
<25\end{array}$ & 5 & Ischemic stroke & 138 \\
\hline $\begin{array}{l}\text { Reitz et al.,29 } \\
2008, \\
\text { Netherlands }\end{array}$ & $\geq 55 \mathrm{yr}$ & $6724(60)$ & 0 & 69.2 & MMSE score $<26$ & 7.3 & All stroke & 713 \\
\hline $\begin{array}{l}\text { Sabayan et al., }{ }^{30} \\
2013, \\
\text { Netherlands }\end{array}$ & $85 \mathrm{yr}$ & $480(66)$ & 0 & 85 & $\begin{array}{l}\text { MMSE score } 25-27 \\
<25\end{array}$ & 5 & All stroke & 56 \\
\hline $\begin{array}{l}\text { Shipley et al. } \text {. }^{31} \\
\text { 2008, United } \\
\text { Kingdom }\end{array}$ & General & $6424(55)$ & NAt & $\begin{array}{l}\text { Range } \\
18-97\end{array}$ & Slow CRT & 21 & Fatal stroke & 170 \\
\hline $\begin{array}{l}\text { Skoog et al..32 } \\
2005, \text { multiple } \\
\text { countries }\end{array}$ & $\begin{array}{l}\text { Hypertension, } \\
70-89 \text { yr }\end{array}$ & $4937(64)$ & 4 & 76 & MMSE score $24-28$ & 3.7 & $\begin{array}{l}\text { All stroke and } \\
\text { ischemic stroke }\end{array}$ & 204 \\
\hline $\begin{array}{l}\text { Weinstein } \\
\text { et al., }{ }^{33} 2013 \text {, } \\
\text { United States }\end{array}$ & General & $1679(53)$ & 0 & 65.7 & $\begin{array}{l}\text { TrB; cognitive } \\
Z \text { scores }<-1.5\end{array}$ & 7.4 & All stroke & 55 \\
\hline $\begin{array}{l}\text { Wiberg et al., }{ }^{34} \\
\text { 2010, Sweden }\end{array}$ & Men, $70 \mathrm{yr}$ & $930(0)$ & 0 & 70 & $\begin{array}{l}\text { MMSE score } 29,28 \\
\leq 27\end{array}$ & 11.1 & All stroke + TIA & 166 \\
\hline $\begin{array}{l}\text { Zhu et al., }{ }^{35} \\
\text { 2000, Sweden }\end{array}$ & $\geq 75 \mathrm{yr}$ & $1551(76)$ & 0 & 82 & $\begin{array}{l}\text { MMSE score < } 24 \\
\text { without dementia; } \\
\text { dementia based on } \\
\text { DSM III criteria }\end{array}$ & 2.6 & All stroke & 110 \\
\hline \multicolumn{9}{|c|}{$\begin{array}{l}\text { Note: } \mathrm{ACHEI}=\text { acetylcholinesterase inhibitors, CIND = cognitive impairment without dementia, CRT = Choice Reaction Time, DSM = Diagnostic and Statistical } \\
\text { Manual of Mental Disorders, DWR }=\text { Delayed Word Recall test, } \mathrm{HAMT}=\text { Hodkinson Abbreviated Mental Test, MMSE }=\text { Mini-Mental State Examination, NA = not } \\
\text { available, SPMSQ }=\text { Short Portable Mental Status Questionnaire, TIA = transient ischemic attack, TrB }=\text { Trail Making Test, part B (executive function performance). } \\
\text { *Random sample from family practitioners' list of all patients. } \\
\text { tRandom sample of community-dwelling adults. }\end{array}$} \\
\hline
\end{tabular}


to the method of Begg and Mazumdar. ${ }^{14}$ We used RevMan 5.2 for the meta-analyses. ${ }^{15}$

The main outcome of interest was the risk of future stroke among patients with cognitive impairment at baseline. Given the various definitions of cognitive impairment across the studies, we took 2 approaches to analyzing the data. First, we combined data from all included studies regardless of the definition used. Second, because one national guideline considers a Mini-Mental State Examination score of 25-30 as normal, ${ }^{16}$ we analyzed results only from studies that used a widely adopted definition of cognitive impairment (i.e., Mini-Mental State Examination score $<25$ or nearest equivalent) and explored the association of this definition of cognitive impairment with future stroke. To clarify whether a history of cognitive impairment gives a clinician extra information about the risk of a stroke beyond what is captured by traditional risk factors for stroke, we conducted an analysis restricted to studies that used maximal adjustment for potential confounders; the studies included in this analysis provided adjustment of all 7 major potential confounders (age, sex, hypertension or systolic blood pressure or antihypertensive drug use, diabetes mellitus, body mass index or other measure of overweight or obesity, cholesterol concentration or statin use, and smoking).

We performed subgroup analyses for cognitive impairment based on data from all included studies according to cognitive scale score (MiniMental State Examination score of 25-29 or nearest equivalent v. $<25$ or nearest equivalent), study design (ordinary cohort v. secondary analysis of clinical trials), study location (North America v. Europe v. international or Asian country), follow-up duration ( $<5 \mathrm{yr}$ v. $\geq 5 \mathrm{yr}$ ), sample size ( $<5000$ vs. $\geq 5000)$, method used to determine cognitive impairment (Mini-Mental State Examination v. other methods), exclusion of people with a history of stroke (excluded $\mathrm{v}$. not excluded) and study quality (high-quality v. low-quality score). We conducted further analyses based on stroke outcome (fatal v. nonfatal) and type of stroke (ischemic v. hemorrhagic).

\section{Results}

\section{Search results and study characteristics}

Of 7518 potentially relevant studies identified through the literature search, 115 were retrieved for detailed assessment. We excluded 87 because

Table 2: Quality assessment of included studies

\begin{tabular}{|c|c|c|c|c|c|c|c|c|}
\hline Study & $\begin{array}{l}\text { Prospective } \\
\text { design }\end{array}$ & $\begin{array}{c}\text { Maintenance } \\
\text { of comparable } \\
\text { groups }\end{array}$ & $\begin{array}{c}\text { Follow-up } \\
\geq 5 \mathrm{yr}\end{array}$ & $\begin{array}{l}\text { Adjustment for } \\
\text { all } 7 \text { potential } \\
\text { confounders* }\end{array}$ & $\begin{array}{l}\text { Baseline } \\
\text { stroke } \\
\text { excluded }\end{array}$ & $\begin{array}{c}\text { Total stroke } \\
\text { reported } t\end{array}$ & $\begin{array}{c}\text { Generalizability } \\
\text { to other } \\
\text { populations }\end{array}$ & $\begin{array}{l}\text { Overall } \\
\text { quality } \\
\text { score }\end{array}$ \\
\hline de Moraes et al. ${ }^{18}$ & Yes & Yes & Yes & No & Yes & No & Yes & 5 \\
\hline Chi et al. ${ }^{19}$ & No & Yes & No & No & Yes & Yes & Yes & 4 \\
\hline Clarke et al. ${ }^{20}$ & Yes & Yes & Yes & No & Yes & Yes & Yes & 6 \\
\hline de Galan et al. ${ }^{21}$ & Yes & Yes & Yes & Yes & No & Yes & No & 5 \\
\hline Ferrucci et al. 22 & Yes & Yes & No & No & No & Yes & Yes & 4 \\
\hline Gale et al. ${ }^{23}$ & Yes & Yes & Yes & No & No & No & Yes & 4 \\
\hline Glymour et al. ${ }^{24}$ & Yes & Yes & Yes & No & Yes & Yes & Yes & 6 \\
\hline Liebetrau et al. ${ }^{25}$ & Yes & Yes & No & Yes & Yes & Yes & No & 5 \\
\hline O'Donnell et al. ${ }^{26}$ & Yes & Yes & No & Yes & No & Yes & No & 4 \\
\hline Ostir et al. ${ }^{27}$ & Yes & Yes & Yes & No & Yes & Yes & Yes & 6 \\
\hline Pettigrew et al. ${ }^{28}$ & Yes & Yes & Yes & No & No & No & No & 3 \\
\hline Reitz et al. ${ }^{29}$ & Yes & Yes & Yes & No & Yes & Yes & Yes & 6 \\
\hline Sabayan et al. ${ }^{30}$ & Yes & Yes & Yes & No & Yes & Yes & No & 5 \\
\hline Shipley et al. ${ }^{31}$ & Yes & Yes & Yes & No & No & No & Yes & 4 \\
\hline Skoog et al. ${ }^{32}$ & Yes & Yes & No & No & No & Yes & No & 3 \\
\hline Weinstein et al. ${ }^{33}$ & Yes & Yes & Yes & No & Yes & Yes & Yes & 6 \\
\hline Wiberg et al. ${ }^{34}$ & Yes & Yes & Yes & No & Yes & Yes & No & 5 \\
\hline Zhu et al. ${ }^{35}$ & Yes & Yes & No & No & Yes & Yes & No & 4 \\
\hline
\end{tabular}


the association of baseline cognitive impairment and future stroke was not reported, 6 because most of the participants had a history of stroke at baseline, 3 because they were duplicate reports and 1 because no adjusted estimate was reported. ${ }^{17}$ Our final primary analysis included 18 cohort studies ${ }^{18-35}$ (Figure 1).

Characteristics of the 18 included studies are shown in Table 1. The total number of participants was 121879 , with 7799 reported stroke events. The studies varied with regard to primary outcome: 12 reported total stroke as a primary outcome, 1 reported fatal and nonfatal stroke separately, 1 reported ischemic and hemorrhagic stroke separately, 1 reported fatal stroke only, 2 reported ischemic stroke only, and 1 reported fatal ischemic stroke only. Transient ischemic attacks were included as outcomes in 2 studies. Participants were derived from ordinary cohorts in 14 studies $^{18-20,22-25,27,29-31,33-35}$ and clinical trials in 4 . $^{21,26,28,32}$

Most of the studies were from North American or European countries. One was conducted in Taiwan, and 3 were an international collaboration. The samples ranged from 401 to 30959 participants, and the follow-up duration ranged

\begin{tabular}{|c|c|c|c|c|c|c|c|}
\hline Study* & Weight, \% & Relative risk $(95 \% \mathrm{Cl})$ & & $\stackrel{\text { Dec }}{\longleftarrow}$ & $\begin{array}{l}\text { ased risk } \\
\text { of stroke }\end{array}$ & $\begin{array}{l}\text { Increased risk } \\
\text { of stroke } \longrightarrow\end{array}$ & \\
\hline de Moraes et al. ${ }^{18}$ & 3.0 & $1.50(1.01-2.23)$ & & & & & \\
\hline Chi et al. ${ }^{19}(a)$ & 4.3 & $1.66(1.40-1.97)$ & & & & $\rightarrow$ & \\
\hline Chi et al. ${ }^{19}(b)$ & 3.1 & $1.70(1.16-2.48)$ & & & & $\rightarrow$ & \\
\hline Clarke et al. ${ }^{20}$ (c) & 3.4 & $2.06(1.50-2.84)$ & & & & $\longrightarrow$ & \\
\hline Clarke et al. ${ }^{20}(\mathrm{~d})$ & 3.8 & $0.42(0.32-0.54)$ & & & $\because$ & & \\
\hline de Galan et al. ${ }^{21}$ (e) & 4.2 & $1.21(1.00-1.47)$ & & & & & \\
\hline de Galan et al. ${ }^{21}$ (f) & 3.0 & $1.32(0.89-1.96)$ & & & & & \\
\hline Ferrucci et al..$^{22}$ (e) & 3.9 & $1.20(0.94-1.54)$ & & & & & \\
\hline Ferrucci et al. ${ }^{22}$ (f) & 2.9 & $2.20(1.45-3.34)$ & & & & & \\
\hline Gale et al. ${ }^{23}(\mathrm{e})$ & 3.5 & $1.30(0.96-1.76)$ & & & & & \\
\hline Gale et al. ${ }^{23}$ (f) & 2.6 & $2.80(1.76-4.46)$ & & & & & \\
\hline Glymour et al. ${ }^{24}$ & 4.5 & $1.26(1.13-1.40)$ & & & & $\rightarrow$ & \\
\hline Liebetrau et al. ${ }^{25}$ & 2.8 & $1.70(1.10-2.63)$ & & & & & \\
\hline O’Donnell et al. ${ }^{26}(\mathrm{e})$ & 4.5 & $1.19(1.05-1.35)$ & & & & & \\
\hline O'Donnell et al. ${ }^{26}(\mathrm{e} 1)$ & 4.3 & $1.30(1.11-1.53)$ & & & & & \\
\hline O'Donnell et al. ${ }^{26}$ (f) & 4.2 & $1.44(1.20-1.73)$ & & & & & \\
\hline Ostir et al. ${ }^{27}$ & 3.6 & $1.85(1.38-2.48)$ & & & & $\longrightarrow$ & \\
\hline Pettigrew et al. ${ }^{28}(\mathrm{e})$ & 3.0 & $1.03(0.70-1.52)$ & & & & & \\
\hline Pettigrew et al. ${ }^{28}(f)$ & 2.2 & $1.63(0.92-2.88)$ & & & & & \\
\hline Reitz et al. ${ }^{29}$ & 3.7 & $1.90(1.43-2.52)$ & & & & & \\
\hline Sabayan et al. ${ }^{30}(\mathrm{e})$ & 2.5 & $1.22(0.74-2.02)$ & & & & & \\
\hline Sabayan et al. ${ }^{30}$ (f) & 2.6 & $2.14(1.32-3.46)$ & & & & & \\
\hline Shipley et al. ${ }^{31}$ & 4.5 & $1.28(1.14-1.44)$ & & & & & \\
\hline Skoog et al. ${ }^{32}$ & 3.9 & $1.20(0.94-1.54)$ & & & & & \\
\hline Weinstein et al. ${ }^{33}$ & 2.5 & $2.25(1.37-3.70)$ & & & & & \\
\hline Wiberg et al. ${ }^{34}(\mathrm{e})$ & 3.0 & $1.05(0.71-1.56)$ & & & & & \\
\hline Wiberg et al. ${ }^{34}(\mathrm{e} 1)$ & 2.8 & $0.83(0.53-1.29)$ & & & & & \\
\hline Wiberg et al. ${ }^{34}(\mathrm{e} 2)$ & 2.6 & $0.62(0.38-1.00)$ & & & & & \\
\hline Zhu et al. ${ }^{35}(\mathrm{e})$ & 2.7 & $2.00(1.26-3.17)$ & & & & & \\
\hline Zhu et al. ${ }^{35}$ (f) & 2.5 & $2.60(1.57-4.30)$ & & & & & \\
\hline Overall & 100.0 & $1.39(1.24-1.56)$ & & & & & \\
\hline \multirow[t]{3}{*}{ Heterogeneity: $I^{2}=82 \%$} & & & $T$ & $T$ & $T$ & $T$ & $T$ \\
\hline & & & 0.1 & 0.2 & 0.5 & $\begin{array}{lll}1 & 2 & 5\end{array}$ & 10 \\
\hline & & & \multicolumn{5}{|c|}{ Relative risk $(95 \% \mathrm{Cl})$} \\
\hline
\end{tabular}

Figure 2: Association between cognitive impairment at baseline and risk of future stroke. Values greater than 1.0 indicate an increased risk of stroke. *Study subgroups: $a=$ ischemic, b= hemorrhagic, $c=$ fatal and $d=$ nonfatal stroke; e, e1, e2, and $f$ represent different degrees of cognitive impairment reported in a study. $\mathrm{Cl}=$ confidence interval. 
from 2.6 to 21 years. All but one of the studies included both men and women; the remaining study included only men. Ten studies used the Mini-Mental State Examination to assess cognitive function, and 8 studies used other cognitive measures. Participants with a history of stroke were excluded in 12 studies, ${ }^{18-20,22,24,25,27,29,30,33-35}$ in the other studies, the proportion of participants with a history of stroke at baseline ranged from $4 \%{ }^{32}$ to $25 \% .{ }^{28}$ Seventeen studies used multivariable-adjusted analysis, and 1 study used propensity score matching. ${ }^{19}$ On a scale of 7 , the overall quality of the studies was good (median score 5, range 3-6) (Table 2).

The funnel plot showed no major asymmetry, and we found no evidence of publication bias using the Begg test $(p=0.1)$ (Appendix 1, available at www.cmaj.ca/lookup/suppl/doi:10.1503/ cmaj.140147/-/DC1).

\section{Primary analysis}

In the primary analysis of pooled data from all of the studies, we found an increased risk of future stroke among patients with cognitive impairment at baseline (relative risk [RR] 1.39, 95\% confidence interval $[\mathrm{CI}]$ 1.24-1.56; random-effects model) (Figure 2). Heterogeneity was high $\left(I^{2}=82 \%\right)$. The estimates were similar between the fixed-effects model (RR 1.31, 95\% CI 1.26$1.37)$ and the random-effects model. In the 3 studies with maximal adjustment for potential confounders, baseline cognitive impairment was associated with increased risk of future stroke (RR 1.28, 95\% CI 1.18-1.38; random-effects model), ${ }^{21,25,26}$ there was no heterogeneity among these studies $\left(I^{2}=0 \%\right)$.

When we pooled results from the studies that used a widely adopted definition of cognitive impairment (i.e., Mini-Mental State Examination score $<25$ or nearest equivalent), the increased risk of future stroke among patients with cognitive impairment at baseline was still evident (RR 1.64, 95\% CI 1.46-1.84; random-effects model) (Figure 3). There was no obvious heterogeneity among these studies $\left(I^{2}=41 \%\right)$. Estimates were similar between the fixed-effects model (RR 1.57, 95\% CI 1.45-1.71) and the random-effects model. In 2 studies with maximal adjustment for potential confounders, baseline cognitive impairment was associated with increased risk of future stroke (RR $1.35,95 \%$ CI 1.21-1.52; random-effect model) ${ }^{22,26}$ there was no heterogeneity among the studies $\left(I^{2}=0 \%\right)$.

A sensitivity analysis of omitting 1 study in each turn did not change the overall results. Another sensitivity analysis, in which we excluded studies that imputed the risk estimates from other stroke outcomes (e.g., ischemic, fatal stroke) if data on total stroke were not available, revealed similar results (RR 1.38, 95\% CI 1.21$1.58 ; 24$ reports from 14 studies; $I^{2}=85 \%$; random-effects model).

\section{Subgroup analyses}

Baseline cognitive impairment was associated with an increased risk of subsequent stroke in all subgroups when we stratified estimates by $\operatorname{cog}$ nitive scale score, study design, study location,

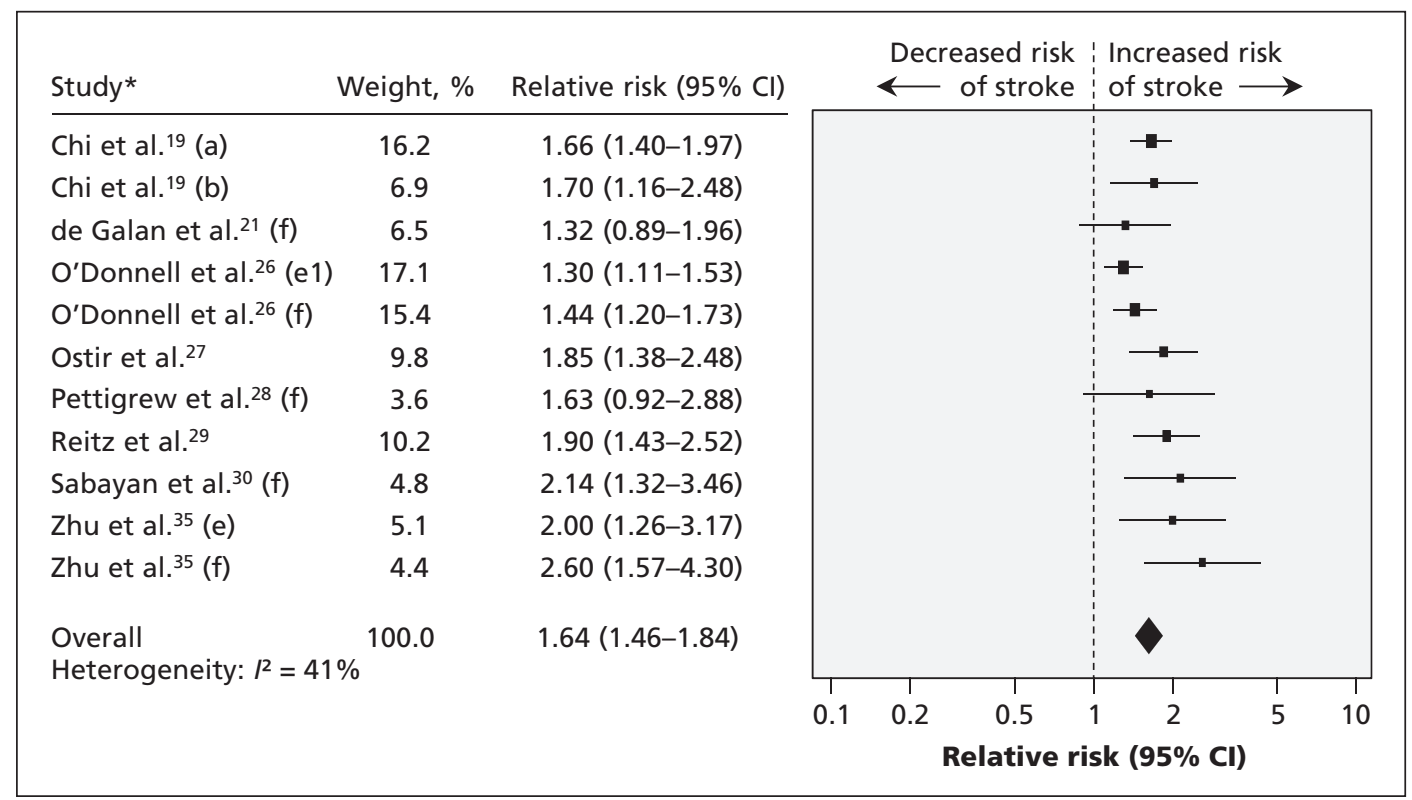

Figure 3: Association between cognitive impairment at baseline defined by Mini-Mental State Examination score $<\mathbf{2 5}$ or nearest equivalent and risk of future stroke. Values greater than $\mathbf{1 . 0}$ indicate an increased risk of future stroke. *Study subgroups: $a=$ ischemic, $b=$ hemorrhagic; e, e1 and $f$ represent different degrees of cognitive impairment reported in a study. $\mathrm{Cl}=$ confidence interval. 
follow-up duration, sample size, method used to determine cognitive impairment, exclusion of people with a history of stroke at baseline, and study quality. We observed significant heterogeneity between pooled analyses for cognitive scale score (Mini-Mental State Examination score $25-29$ or nearest equivalent v. $<25$ or nearest equivalent: RR $1.16,95 \%$ CI $1.06-1.27$ v. RR $1.64,95 \%$ CI $1.46-1.84 ; l^{2}=95 \%$ ); there was no substantial heterogeneity within each category of cognitive scale score. No obvious heterogeneity was found between other characteristics of participants (Table 3 ).

When we conducted further analyses based on stroke outcome (fatal v. nonfatal) and type of stroke (ischemic v. hemorrhagic), we found that baseline cognitive impairment was associated with an increased risk of fatal stroke (RR 1.68, 95\% CI 1.21-2.33; 3 studies; $I^{2}=82 \%$; randomeffects model) but not with nonfatal stroke (RR $0.71,95 \%$ CI $0.25-2.01 ; 2$ studies; $I^{2}=97 \%$; random-effects model) (Figure 4). When we pooled the data by ischemic or hemorrhagic stroke, we found an association between baseline cognitive impairment and an increased risk of ischemic stroke (RR 1.65, 95\% CI 1.41-1.93; 4 studies; $P^{2}=21 \%$; random-effects model) but no increased risk of hemorrhagic stroke (RR 0.57, 95\% CI 0.20-1.57; 2 studies; $I^{2}=92 \%$ ) (Figure 4).

\section{Interpretation}

In this meta-analysis of 18 cohort studies involving more than 120000 people and almost 8000

\begin{tabular}{|c|c|c|c|c|}
\hline Subgroup & $\begin{array}{l}\text { No. of } \\
\text { studies }\end{array}$ & $\begin{array}{l}\text { Relative risk } \\
\quad(95 \% \mathrm{Cl})\end{array}$ & $\begin{array}{l}\text { Heterogeneity } \\
\text { within subgroups }\end{array}$ & $\begin{array}{l}\text { Heterogeneity } \\
\text { among subgroups }\end{array}$ \\
\hline Cognitive scale score & & & & $p<0.001, l^{2}=95 \%$ \\
\hline $\begin{array}{l}\text { MMSE } 25-29 \text { or nearest } \\
\text { equivalent }\end{array}$ & 6 & $1.16(1.06-1.27)$ & $p=0.8, p^{2}=0 \%$ & \\
\hline $\begin{array}{l}\text { MMSE }<25 \text { or nearest } \\
\text { equivalent }\end{array}$ & 8 & $1.64(1.46-1.84)$ & $p=0.08, l^{2}=41 \%$ & \\
\hline Study design & & & & $p=0.1, I^{2}=62 \%$ \\
\hline Ordinary cohorts & 14 & $1.46(1.24-1.72)$ & $p<0.001, R^{2}=86 \%$ & \\
\hline Analysis of trials & 4 & $1.26(1.17-1.35)$ & $p=0.6, R^{2}=0 \%$ & \\
\hline Sample size & & & & $p=0.5, l^{2}=0 \%$ \\
\hline$\geq 5000$ & 9 & $1.34(1.16-1.55)$ & $p<0.001, R^{2}=87 \%$ & \\
\hline$<5000$ & 9 & $1.47(1.20-1.80)$ & $p<0.001, R^{2}=72 \%$ & \\
\hline Follow-up duration, yr & & & & $p=0.3, R^{2}=17 \%$ \\
\hline$\geq 5$ & 12 & $1.32(1.11-1.57)$ & $p<0.001, R^{2}=86 \%$ & \\
\hline$<5$ & 6 & $1.48(1.31-1.68)$ & $p=0.002, R^{2}=64 \%$ & \\
\hline Assessment tool & & & & $p=0.8, I^{2}=0 \%$ \\
\hline MMSE & 10 & $1.38(1.23-1.55)$ & $p<0.001, R^{2}=67 \%$ & \\
\hline Other & 8 & $1.43(1.13-1.82)$ & $p<0.001, R^{2}=90 \%$ & \\
\hline Study location & & & & $p=0.8, I^{2}=0 \%$ \\
\hline North America & 7 & $1.38(1.02-1.87)$ & $p<0.001, R^{2}=91 \%$ & \\
\hline Europe & 7 & $1.47(1.19-1.81)$ & $p<0.001, R^{2}=76 \%$ & \\
\hline $\begin{array}{l}\text { Multiple countries } \\
\text { or Asian country }\end{array}$ & 4 & $1.34(1.22-1.48)$ & $p=0.07, R^{2}=47 \%$ & \\
\hline People with prior stroke & & & & $p=0.4, I^{2}=0 \%$ \\
\hline Excluded & 12 & $1.44(1.18-1.76)$ & $p<0.001, R^{2}=88 \%$ & \\
\hline Not excluded & 6 & $1.30(1.19-1.41)$ & $p=0.1, R^{2}=38 \%$ & \\
\hline Study quality & & & & $p=0.4, I^{2}=0 \%$ \\
\hline High, score 5-7 & 10 & $1.29(1.03-1.62)$ & $p<0.001, I^{2}=88 \%$ & \\
\hline Low, score $<5$ & 8 & $1.45(1.30-1.62)$ & $p<0.001, R^{2}=65 \%$ & \\
\hline
\end{tabular}


stroke events, we found that the risk of future stroke was 39\% higher among patients with cognitive impairment at baseline than among those with normal cognitive function at baseline. This risk increased to $64 \%$ when a broadly adopted definition of cognitive impairment was used. This association was consistent across diverse population subgroups. The size and inclusion of mostly prospectively collected data strengthened the robustness of our findings, because selection bias, recall bias and reverse causality were unlikely. In addition, all of the included studies reported multivariable adjusted RRs, which probably mitigated the possibility of known confounders influencing our results.
Differing degrees of cognitive impairment may account for the heterogeneity in the main analysis. In a subgroup analysis, we found a possible dose-response relation between cognitive impairment and stroke: among participants with cognitive impairment, the risk of stroke was significantly greater with a low Mini-Mental State Examination score than with a high score. Heterogeneity was removed by division into strata.

Cognitive impairment may contribute to future stroke through a variety of mechanisms. First, the condition is associated with silent brain infarcts, ${ }^{36,37}$ which increase the risk of subsequent overt stroke. Silent brain infarcts may have been more frequent in the participants with

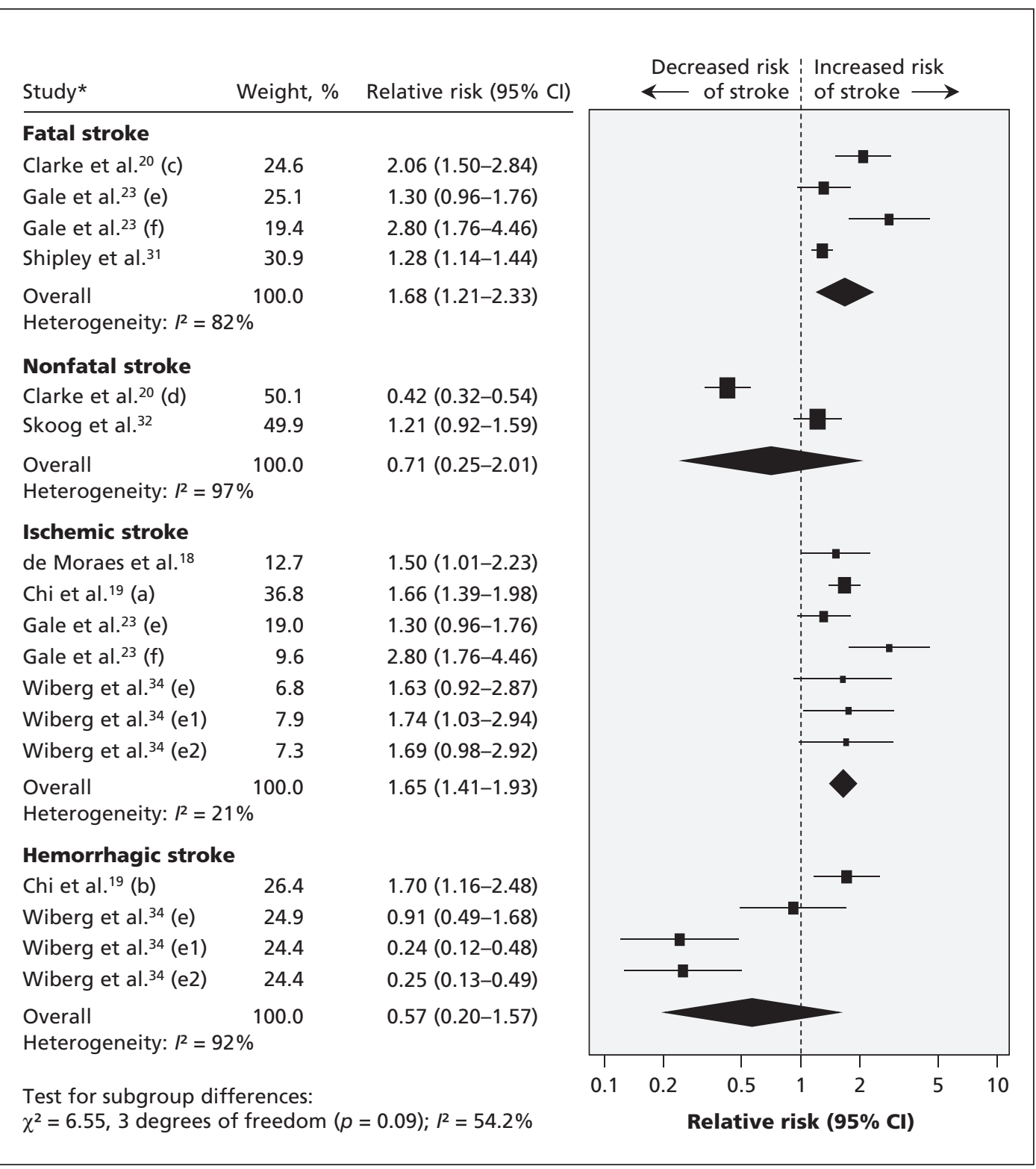

Figure 4: Association between cognitive impairment at baseline and risk of future stroke, by type of stroke. Values greater than $\mathbf{1 . 0}$ indicate an increased risk of stroke. *Study subgroups: $a=$ ischemic, $b=$ hemorrhagic, $c=$ fatal and $d=$ nonfatal stroke; e and $f$ represent different degrees of cognitive impairment reported in a study. $\mathrm{Cl}=$ confidence interval. 
cognitive impairment, but brain imaging was not done at baseline in most of the studies in our meta-analysis. Also, people with cognitive impairment tend to have white-matter hyperintensities, ${ }^{38}$ disturbances of cerebrovascular hemodynamics, ${ }^{39}$ deposition of amyloid in cerebral vessels ${ }^{6}$ and microbleeds, ${ }^{40,41}$ which may in turn increase the risk of future stroke. Most dementias are contributed to by vascular disease, and as such, patients who experience multiple small white-matter infarcts may go on to have dementia without ever having traditional stroke symptoms. The only way to assess these lesions is through magnetic resonance imaging, which was not performed in the studies included in our study. It is thus not inconceivable that dementia may be an epiphenomenon of prior vascular disease, and established vascular disease by itself will beget more strokes. Second, previous studies have shown that several biomarkers of systemic atherosclerosis and inflammation, such as elevated homocysteine and C-reactive protein, are associated with an increased risk of both cognitive impairment and stroke. ${ }^{42-44}$ Also, increased coronary artery calcification is associated with poor memory in midlife ${ }^{45}$ and independently increases the risk of future stroke in the general population. ${ }^{46}$ Third, cognitive impairment is associated with high within-individual variability in blood pressure, ${ }^{47}$ which itself is a risk factor for stroke. ${ }^{48,49}$ Finally, cognitive impairment can be linked to various deleterious factors (e.g., lack of medication compliance, poor diet, physical inactivity, frailty and depression), which increase the risk of stroke..$^{50-54}$

The impact of stroke on future dementia seems greater than the impact of cognitive impairment on future stroke. A Canadian study suggested that the presence of both stroke and $\mathrm{APOE}_{4}$ genotype compared with the absence of these 2 factors was associated with a greater risk of dementia (RR 2.57). ${ }^{55}$ Screening for cognitive impairment has been recommended in patients with stroke, given results from a recent Canadian study showing that two-thirds of stroke patients have evidence for cognitive impairment. ${ }^{56}$ Our findings suggest that identifying people with cognitive impairment may provide an even bigger opportunity to reduce the future burden of stroke through the timely implementation of evidence-based prevention strategies.

\section{Limitations}

Our study has limitations. First, meta-analyses may be biased if the literature search fails to identify all relevant studies or the selection criteria for including a study are applied in a subjective manner. To minimize these risks, we car- ried out thorough searches across different databases using explicit criteria for study selection, data abstraction and data analysis.

Second, we observed a large amount of heterogeneity in the results among all included studies. However, there was no significant heterogeneity among studies that provided information using a widely adopted definition of cognitive impairment.

Third, the sensitivity of the Mini-Mental State Examination to detect cognitive impairment has been found to be moderate compared with the Montreal Cognitive Assessment. ${ }^{57}$ Also, different degrees of cognitive impairment may have different effects on the risk of future stroke. Because most of the included studies did not provide a detailed description of the characteristics of the cognitive dysfunction, we were unable to explore this issue further.

Fourth, it would have been better to analyze sex-specific differences in the risk of stroke. However, our study was a study-level metaanalysis, and almost all of the included studies did not provide such data. Pooled analysis of patient-level data from relevant studies is warranted and may provide additional insights.

Finally, the association of cognitive impairment and risk of hemorrhagic stroke was inconclusive, because only 2 studies provided such information, and heterogeneity was high. Because cognitive impairment and intracranial hemorrhage share some pathophysiologic mechanisms, such as cerebral amyloid angiopathy, ${ }^{6,58,59}$ further studies are warranted to clarify this issue.

\section{Conclusion}

We found that the risk of future stroke was increased among patients with cognitive impairment at baseline, particularly among those who met a broadly adopted clinical definition of cognitive impairment. Cognitive impairment should be more broadly recognized as a possible early clinical manifestation of cerebral infarction, so that timely management of vascular risk factors can be instituted to potentially prevent future stroke events and to avoid further deterioration of cognitive health.

\section{References}

1. Donnan GA, Fisher M, Macleod M, et al. Stroke. Lancet 2008; 371:1612-23.

2. Seshadri S, Beiser A, Kelly-Hayes M, et al. The lifetime risk of stroke: estimates from the Framingham Study. Stroke 2006;37: 345-50.

3. Hachinski V, Iadecola C, Petersen RC, et al. National Institute of Neurological Disorders and Stroke-Canadian Stroke Network vascular cognitive impairment harmonization standards [published erratum in Stroke 2007;38:1118]. Stroke 2006;37:2220-41.

4. Ovbiagele B, Goldstein LB, Higashida RT, et al. Forecasting the future of stroke in the United States: a policy statement from the American Heart Association and American Stroke Association. Stroke 2013;44:2361-75. 
5. Evans DA, Funkenstein HH, Albert MS, et al. Prevalence of Alzheimer's disease in a community population of older persons. Higher than previously reported. JAMA 1989;262:2551-6.

6. Kalaria RN. Linking cerebrovascular defense mechanisms in brain ageing and Alzheimer's disease. Neurobiol Aging 2009;30: 1512-4.

7. Polidori MC, Pientka L, Mecocci P. A review of the major vascular risk factors related to Alzheimer's disease. J Alzheimers Dis 2012;32:521-30

8. Dregan A, Wolfe CD, Gulliford MC. Does the influence of stroke on dementia vary by different levels of prestroke cognitive functioning? A cohort study. Stroke 2013;44:3445-51.

9. Stroup DF, Berlin JA, Morton SC, et al. Meta-analysis of observational studies in epidemiology: a proposal for reporting. Meta-analysis Of Observational Studies in Epidemiology (MOOSE) group. JAMA 2000;283:2008-12.

10. Pan A, Sun Q, Okereke OI, et al. Depression and risk of stroke morbidity and mortality: a meta-analysis and systematic review. JAMA 2011;306:1241-9.

11. Higgins JP, Thompson SG. Quantifying heterogeneity in a meta-analysis. Stat Med 2002;21:1539-58.

12. Higgins JP, Thompson SG, Deeks JJ, et al. Measuring inconsistency in meta-analyses. BMJ 2003;327:557-60.

13. Higgins JPT, Green S. Cochrane handbook for systematic reviews of interventions: Oxford (UK): Cochrane Collaboration; 2008.

14. Begg CB, Mazumdar M. Operating characteristics of a rank correlation test for publication bias. Biometrics 1994;50:1088-101.

15. Lee M, Saver JL, Chang KH, et al. Low glomerular filtration rate and risk of stroke: meta-analysis. BMJ 2010;341:c4249.

16. Dementia: supporting people with dementia and their careers in health and social care [clinical guideline]. London (UK): NICE; 2006. Available: http://guidance.nice.org.uk/CG42 (accessed 2013 Nov. 6)

17. Imfeld P, Bodmer M, Schuerch M, et al. Risk of incident stroke in patients with Alzheimer disease or vascular dementia. Neurology 2013;81:910-9.

18. de Moraes SA, Szklo M, Tilling K, et al. Cognitive functioning as a predictor of ischemic stroke incidence. Epidemiology 2003;14:673-9.

19. Chi NF, Chien LN, Ku HL, et al. Alzheimer disease and risk of stroke: a population-based cohort study. Neurology 2013;80: 705-11.

20. Clarke PJ, Blount V, Colantonio A. Cognitive impairment predicts fatal incident stroke: findings from a national sample of older adults. J Am Geriatr Soc 2011;59:1490-6.

21. de Galan BE, Zoungas S, Chalmers J, et al. Cognitive function and risks of cardiovascular disease and hypoglycaemia in patients with type 2 diabetes: the Action in Diabetes and Vascular Disease: Preterax and Diamicron Modified Release Controlled Evaluation (ADVANCE) trial. Diabetologia 2009;52:2328-36.

22. Ferrucci L, Guralnik JM, Salive ME, et al. Cognitive impairmen and risk of stroke in the older population. J Am Geriatr Soc 1996; 44:237-41.

23. Gale CR, Martyn CN, Cooper C. Cognitive impairment and mortality in a cohort of elderly people. BMJ 1996;312:608-11.

24. Glymour MM, Maselko J, Gilman SE, et al. Depressive symptoms predict incident stroke independently of memory impairments. Neurology 2010;75:2063-70.

25. Liebetrau M, Steen B, Skoog I. Depression as a risk factor for the incidence of first-ever stroke in 85-year-olds. Stroke 2008; 39:1960-5

26. O'Donnell M, Teo K, Gao P, et al. Cognitive impairment and risk of cardiovascular events and mortality. Eur Heart J 2012; 33:1777-86.

27. Ostir GV, Raji MA, Ottenbacher KJ, et al. Cognitive function and incidence of stroke in older Mexican Americans. J Geronto A Biol Sci Med Sci 2003;58:531-5.

28. Pettigrew LC, Thomas N, Howard VJ, et al. Low mini-menta status predicts mortality in asymptomatic carotid arterial stenosis. Asymptomatic Carotid Atherosclerosis Study investigators. Neurology 2000;55:30-4.

29. Reitz C, Bos MJ, Hofman A, et al. Prestroke cognitive performance, incident stroke, and risk of dementia: the Rotterdam Study. Stroke 2008;39:36-41.

30. Sabayan B, Gussekloo J, de Ruijter W, et al. Framingham stroke risk score and cognitive impairment for predicting firsttime stroke in the oldest old. Stroke 2013;44:1866-71.

31. Shipley BA, Der G, Taylor MD, et al. Cognition and mortality from the major causes of death: the Health and Lifestyle Survey. J Psychosom Res 2008;65:143-52.

32. Skoog I, Lithell H, Hansson L, et al. Effect of baseline cognitive function and antihypertensive treatment on cognitive and cardiovascular outcomes: Study on COgnition and Prognosis in the Elderly (SCOPE). Am J Hypertens 2005;18:1052-9.
33. Weinstein G, Beiser AS, Decarli C, et al. Brain imaging and cognitive predictors of stroke and Alzheimer disease in the Framingham Heart Study. Stroke 2013;44:2787-94.

34. Wiberg B, Lind L, Kilander L, et al. Cognitive function and risk of stroke in elderly men. Neurology 2010;74:379-85.

35. Zhu L, Fratiglioni L, Guo Z, et al. Incidence of stroke in relation to cognitive function and dementia in the Kungsholmen Project. Neurology 2000;54:2103-7.

36. Kuller LH, Shemanski L, Manolio T, et al. Relationship between ApoE, MRI findings, and cognitive function in the Cardiovascular Health Study. Stroke 1998;29:388-98.

37. Vermeer SE, Prins ND, den Heijer T, et al. Silent brain infarcts and the risk of dementia and cognitive decline. $N$ Engl J Med 2003; 348:1215-22.

38. Luchsinger JA, Brickman AM, Reitz C, et al. Subclinical cerebrovascular disease in mild cognitive impairment. Neurology 2009;73:450-6.

39. Sabayan B, Jansen S, Oleksik AM, et al. Cerebrovascular hemodynamics in Alzheimer's disease and vascular dementia: a meta-analysis of transcranial Doppler studies. Ageing Res Rev 2012;11:271-7.

40. van Norden AG, van Uden IW, de Laat KF, et al. Cerebral microbleeds are related to subjective cognitive failures: the RUN DMC study. Neurobiol Aging 2013;34:2225-30.

41. Park JH, Seo SW, Kim C, et al. Pathogenesis of cerebral microbleeds: In vivo imaging of amyloid and subcortical ischemic small vessel disease in 226 individuals with cognitive impairment. Ann Neurol 2013;73:584-93.

42. Homocysteine Studies Collaboration. Homocysteine and risk of ischemic heart disease and stroke: a meta-analysis. JAMA 2002;288:2015-22.

43. Kuo HK, Yen CJ, Chang CH, et al. Relation of C-reactive protein to stroke, cognitive disorders, and depression in the general population: systematic review and meta-analysis. Lancet Neurol 2005;4:371-80

44. Feng L, Isaac V, Sim S, et al. Associations between elevated homocysteine, cognitive impairment, and reduced white matte volume in healthy old adults. Am J Geriatr Psychiatry 2013;21: 164-72.

45. Reis JP, Launer LJ, Terry JG, et al. Subclinical atherosclerotic calcification and cognitive functioning in middle-aged adults: The CARDIA study. Atherosclerosis 2013;231:72-7.

46. Hermann DM, Gronewold J, Lehmann N, et al. Coronary artery calcification is an independent stroke predictor in the general population. Stroke 2013;44:1008-13.

47. Sabayan B, Wijsman LW, Foster-Dingley JC, et al. Association of visit-to-visit variability in blood pressure with cognitive function in old age: prospective cohort study. BMJ 2013;347: f4600.

48. Hashimoto T, Kikuya M, Ohkubo T, et al. Home blood pressure level, blood pressure variability, smoking, and stroke risk in Japanese men: the Ohasama study. Am J Hypertens 2012;25: 883-91.

49. Rothwell PM, Howard SC, Dolan E, et al. Effects of beta blockers and calcium-channel blockers on within-individual variability in blood pressure and risk of stroke. Lancet Neurol 2010;9:469-80

50. Kim G, Kim H, Kim KN, et al. Relationship of cognitive function with B vitamin status, homocysteine, and tissue factor pathway inhibitor in cognitively impaired elderly: a cross-sectional survey. J Alzheimers Dis 2013;33:853-62.

51. Wu MS, Lan TH, Chen CM, et al. Socio-demographic and health-related factors associated with cognitive impairment in the elderly in Taiwan. BMC Public Health 2011;11:22.

52. Campbell NL, Boustani MA, Skopelja EN, et al. Medication adherence in older adults with cognitive impairment: a systematic evidence-based review. Am J Geriatr Pharmacother 2012; 10:165-77.

53. Song X, Mitnitski A, Rockwood K. Nontraditional risk factor combine to predict Alzheimer disease and dementia. Neurology 2011;77:227-34.

54. Richard E, Reitz C, Honig LH, et al. Late-life depression, mild cognitive impairment, and dementia. JAMA Neurol 2013;70: 374-82

55. Jin YP, Ostbye T, Feightner JW, et al. Joint effect of stroke and APOE 4 on dementia risk: the Canadian Study of Health and Aging. Neurology 2008;70:9-16

56. Mai LM, Oczkowski W, Mackenzie G, et al. Screening for cognitive impairment in a stroke prevention clinic using the MoCA. Can J Neurol Sci 2013;40:192-7.

57. Godefroy O, Fickl A, Roussel M, et al. Is the Montreal Cognitive Assessment superior to the Mini-Mental State Examination to detect poststroke cognitive impairment? A study with neuropsychological evaluation. Stroke 2011;42:1712-6. 
58. Tanskanen M, Kalaria RN, Notkola IL, et al. Relationships between white matter hyperintensities, cerebral amyloid angiopathy and dementia in a population-based sample of the oldest old. Curr Alzheimer Res 2013;10:1090-7.

59. Gurol ME, Dierksen G, Betensky R, et al. Predicting sites of new hemorrhage with amyloid imaging in cerebral amyloid angiopathy. Neurology 2012;79:320-6.

Affiliations: Department of Neurology (Lee, Wu), Chang Gung University College of Medicine, Chang Gung Memorial Hospital, Chiayi, Taiwan; Stroke Center and Department of Neurology (Saver, Rao), Geffen School of Medicine, University of California, Los Angeles; Department of Neurology (Hong), Ilsan Paik Hospital, Inje University, Gimhae, South Korea; Department of Psychiatry (Liu), Taipei City Hospital Song-Te Branch, Taipei, Taiwan; Department of Neurosciences (Ovbiagele), Medical University of South Carolina, Charleston, SC
Contributors: Meng Lee, Jeffrey Saver, Hsing-Cheng Liu and Bruce Ovbiagele were involved in the study concept and design. Meng Lee, Keun-Sik Hong and Yi-Ling Wu acquired the data. Meng Lee, Yi-Lin Wu, Neal Rao and Bruce Ovbiagele analyzed and interpreted the data. Meng Lee and Yi-Ling Wu drafted the manuscript. Meng Lee, Jeffrey Saver, Keun-Sik Hong, Hsin-Cheng Liu, Neal Rao and Bruce Ovbiagele revised the manuscript critically for important intellectual content. All of the authors approved the final version of the manuscript submitted for publication and agreed to act as guarantors of the work.

Funding: This study was supported by grants from the Chang Gung Memorial Hospital and the National Science Council, Taiwan (grant nos. CMRPG6B0111, 6B0112 and NSC 102-2628-B-182-012). The sponsors played no role in the study design, data collection and analysis, or decision to submit the article for publication. 